\title{
Serologic Evidence for MERS-CoV Infection in Dromedary Camels, Punjab, Pakistan, 2012-2015
}

\section{Muhammad Saqib, ${ }^{1}$ Andrea Sieberg, ${ }^{1}$ Muhammad Hammad Hussain, Muhammad Khalid Mansoor, Ali Zohaib, Erik Lattwein, Marcel Alexander Müller, Christian Drosten, Victor Max Corman}

Author affiliations: Department of Clinical Medicine and Surgery, University of Agriculture, Faisalabad, Pakistan (M. Saqib, M.H. Hussain); University of Bonn Medical Centre, Bonn, Germany (A. Sieberg, M.A. Müller, C. Drosten, V.M. Corman); Animal Health Research Center, Ministry of Agriculture and Fisheries, Muscat, Oman (M.H. Hussain, M.K. Mansoor); Key Laboratory of Special Pathogens and Biosafety, Wuhan Institute of Virology, Chinese Academy of Sciences, Wuhan, China (A. Zohaib); EUROIMMUN AG, Lübeck, Germany (E. Lattwein); German Centre for Infection Research, Bonn (C. Drosten, V.M. Corman)

DOI: http://dx.doi.org/10.3201/eid2303.161285

Dromedary camels from Africa and Arabia are an established source for zoonotic Middle East respiratory syndrome coronavirus (MERS-CoV) infection among humans. In Pakistan, we found specific neutralizing antibodies in samples from $39.5 \%$ of 565 dromedaries, documenting significant expansion of the enzootic range of MERS-CoV to Asia.

$\mathrm{T}$ he Middle East respiratory syndrome coronavirus (MERS-CoV) is a zoonotic pathogen that causes severe respiratory disease in humans. Dromedary camels (Camelus dromedarius), which have 1 hump on their backs, have been identified as an animal reservoir and source of human MERS-CoV infection (1). Reports document widespread infection of these camels on the Arabian Peninsula and parts of Africa (2-4). Besides these regions, dromedaries are also native to several countries in Asia. A study in Kazakhstan found no evidence for MERS-CoV infection in dromedaries (5). Absence of MERS-CoV infection in dromedary camels in Asia could mean a vulnerable animal reservoir at risk for de novo introduction by sporadic contact (e.g., by trade) with dromedaries from MERS-CoV endemic areas. Pakistan is 1 of 8 countries globally, and the only country outside Africa, that has a dromedary population exceeding 1 million animals (FAOSTAT database; http://faostat3.fao.org). Because of the limited capacities for routine MERS-CoV surveillance and a considerable

${ }^{1}$ These authors contributed equally to this article. human population size in countries in northeastern Asia, targeted investigation of the MERS-CoV infection status is of interest for global public health agencies.

In this study, we examined dromedaries from Pakistan for exposure to MERS-CoV. We tested 565 serum samples, which we collected from 348 female and 217 male animals by using a convenience sampling strategy in 9 districts of Punjab, eastern Pakistan, during 2012-2015. The median age of the animals was 5 years.

The testing algorithm comprised 2 antibody detection methods $(3,6)$. MERS-CoV IgG was detected by using a MERS-CoV camel antibody ELISA (EUROIMMUN, Lübeck, Germany). We tested all serum samples that exceeded a cutoff of 0.4 , validated in previous studies $(3,6)$, by using a microneutralization (MN) test for confirmation (3). Only serum samples with neutralizing activity $\geq 1: 80$ were considered MERS-CoV antibody-positive.

A total of $315(55.8 \%)$ of 565 camel serum samples exceeded the ELISA signal cutoff (Table). Of these, 223 (39.5\%) were confirmed by using MN. We identified MERS-CoV neutralizing antibodies in camels sampled in all study years and in nearly all regions except the district of Chiniot (Table). The rate of neutralizing antibody-positive camel samples ranged from $82.9 \%$ in Rahim Yar Khan to $24.1 \%$ in the Jhang district (Table).

By using a merged dataset comprising all regions, we correlated seropositivity to animal age and sex. Sex-dependent differences suggested pronounced seropositivity in male animals, but differences were not significant ( $\mathrm{p}>0.067$; online Technical Appendix Table, https://wwwnc.cdc.gov/ EID/article/23/3/16-1285-Techapp1.pdf). Seropositivity increased with age: samples from more than half $(51.1 \%)$ of all animals $>5$ years of age and less than one third (29.2\%) of animals $<2$ years of age tested positive $(\mathrm{p}<0.001$; online Technical Appendix Table). These age-dependent differences are similar to those found in previous studies $(6,7)$ and can be explained by long-lasting immune response or regular reexposure after initial MERS-CoV infection. The finding of antibodies in young camels born in Pakistan suggests ongoing circulation of MERS-CoV in the country.

Bactrian camels (C. bactrianus), which have 2 humps on their backs, are also native to Central and East Asia. Studies of these camels in China and Mongolia, as well as dromedary and Bactrian camels in southern Kazakhstan, uniformly reported absence of MERS-CoV during 2014-2015 (5,8,9). However, dromedaries may become sources of infection for Bactrian camels that are susceptible to MERS-CoV and present in this vast geographic range. The possibility of cross-species transmission within the order of camelids has been documented by a study that found signs of MERS-CoV infection in alpacas (Vicugna pacos) that shared a barn with dromedaries in Qatar (10). The absence of MERS-CoV in camelid populations in northeastern parts of Asia (Mongolia, 
Table. Detection of Middle East respiratory syndrome coronavirus antibodies in dromedary camels from different districts of Punjab Province, Pakistan, 2012-2015*

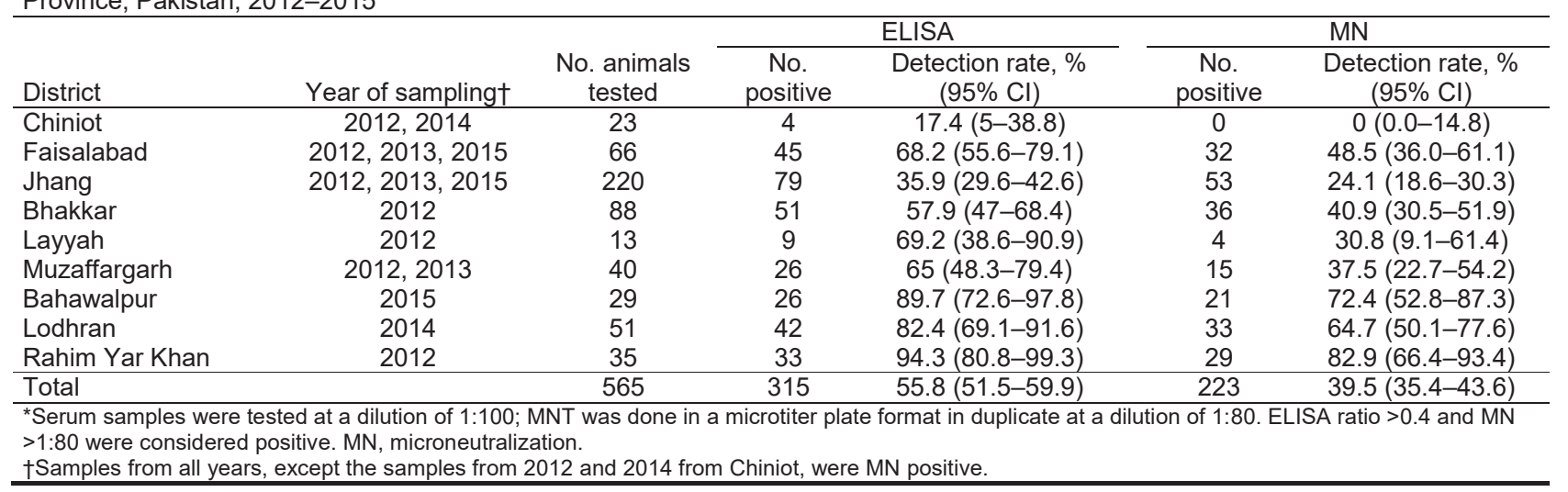

Kazakhstan, and China) is compatible with the view that the spread of MERS-CoV from Africa into Asia may be a recent development. However, other explanations, including resistance to infection by Bactrian camels, are possible. Studies of susceptibility should be conducted to clarify whether Bactrian camels in Asia could act as a naïve reservoir population in the future.

Based on MERS-CoV antibodies in dromedary camels, our data suggest a risk for human exposure in Punjab, Pakistan, that is similar to risks in Africa and the Arabian Peninsula. Of note, Punjab shares a border with the state of Rajasthan in India, which harbors that country's largest dromedary population. A similar risk for human exposure is likely for this part of India. However, findings of antibodies against MERS-CoV in migrant workers from these areas should be interpreted with caution because these workers often employed in Arabian countries. For Pakistan, our data largely exclude the scenario of a widely susceptible animal reservoir population in which de novo introduction of MERS-CoV could start an epizootic that could lead to spillover epidemics among humans.

\section{Acknowledgments}

We thank Tobias Bleicker, Monika Eschbach-Bludau and Sebastian Brünink for excellent technical assistance.

This work was supported by the German Research Foundation (Deutsche Forschungsgemeinschaft grant DR772/12-1 to CD); the European Commission project PREPARE (contract number 602525) and the ZAPI project (http://zapi-imi.eu); Innovative Medicines Initiative (IMI) grant agreement 115760), with the assistance and financial support of IMI and the European Commission; and in-kind contributions from European Federation of Pharmaceutical Industries and Associations partners.

Dr. Saqib is an assistant professor in the field of veterinary medicine, Faculty of Veterinary Science, University of Agriculture, Faisalabad. His main research interests are zoonotic and infectious diseases of domestic and zoo animals.

\section{References}

1. Memish ZA, Cotten M, Meyer B, Watson SJ, Alsahafi AJ, Al Rabeeah AA, et al. Human infection with MERS coronavirus after exposure to infected camels, Saudi Arabia, 2013. Emerg Infect Dis. 2014;20:1012-5. http://dx.doi.org/10.3201/eid2006.140402

2. Reusken CB, Messadi L, Feyisa A, Ularamu H, Godeke GJ, Danmarwa A, et al. Geographic distribution of MERS coronavirus among dromedary camels, Africa. Emerg Infect Dis. 2014;20:1370-4. http://dx.doi.org/10.3201/eid2008.140590

3. Müller MA, Corman VM, Jores J, Meyer B, Younan M, Liljander A, et al. MERS coronavirus neutralizing antibodies in camels, Eastern Africa, 1983-1997. Emerg Infect Dis. 2014;20:2093-5. http://dx.doi.org/10.3201/eid2012.141026

4. Food and Agriculture Organization of the United Nations. MERS-CoV situation update 20 July 2016. 2016 [cited 28 July 2016]. http://www.fao.org/ag/againfo/programmes/en/empres/mers/ situation_update.html

5. Miguel E, Perera RA, Baubekova A, Chevalier V, Faye B, Akhmetsadykov N, et al. Absence of Middle East respiratory syndrome coronavirus in camelids, Kazakhstan, 2015. Emerg Infect Dis. 2016;22:555-7. http://dx.doi.org/10.3201/eid2203.151284

6. Corman VM, Jores J, Meyer B, Younan M, Liljander A, Said MY, et al. Antibodies against MERS coronavirus in dromedary camels, Kenya, 1992-2013. Emerg Infect Dis. 2014;20:1319-22. http://dx.doi.org/10.3201/eid2008.140596

7. Wernery U, Corman VM, Wong EY, Tsang AK, Muth D, Lau SK, et al. Acute Middle East respiratory syndrome coronavirus infection in livestock dromedaries, Dubai, 2014. Emerg Infect Dis. 2015;21:1019-22. http://dx.doi.org/10.3201/eid2106.150038

8. Chan SM, Damdinjav B, Perera RA, Chu DK, Khishgee B, Enkhbold B, et al. Absence of MERS-coronavirus in Bactrian camels, Southern Mongolia, November 2014. Emerg Infect Dis. 2015;21:1269-71. http://dx.doi.org/10.3201/eid2107.150178

9. Liu R, Wen Z, Wang J, Ge J, Chen H, Bu Z. Absence of Middle East respiratory syndrome coronavirus in Bactrian camels in the West Inner Mongolia Autonomous Region of China: surveillance study results from July 2015. Emerg Microbes Infect. 2015;4:e73. http://dx.doi.org/10.1038/emi.2015.73

10. Reusken CB, Schilp C, Raj VS, De Bruin E, Kohl RH, Farag EA, et al. MERS-CoV infection of alpaca in a region where MERS-CoV is endemic. Emerg Infect Dis. 2016;22:1129-31. http://dx.doi.org/10.3201/eid2206.152113

Address for correspondence: Christian Drosten, Institute of Virology, University of Bonn Medical Centre, Sigmund-Freud-Str. 25, 53105 Bonn, Germany; email: drosten@virology-bonn.de 